\title{
Detecting Dual Superconductivity in the Ground State of Gauge Theory.
}

\author{
L. Del Debbio, A. Di Giacomo and G. Paffuti \\ Dipartimento di Fisica dell'Università and I.N.F.N., I-56126 Pisa, Italy
}

\begin{abstract}
We explicitly construct a monopole creation operator: its vacuum expectation value is an order parameter for dual superconductivity, in that, if different from zero, it signals a spontaneous breaking of the $U(1)$ symmetry corresponding to monopole charge conservation.

This operator is tested by numerical simulations in compact $U(1)$ gauge theory. Our construction provides a general recipe for detection of the condensation of any topological soliton. In particular our operator can be used to detect dual superconductivity of the QCD vacuum.
\end{abstract}

PACS numbers: 11.15 Ha, 12.38 Aw, 64.60 Cn

Typeset Using REVTEX 


\section{INTRODUCTION}

A possible mechanism of colour confinement in Quantum Chromo-Dynamics $(Q C D)$ is dual superconductivity of the vacuum [1] 4 .

According to this scenario, the chromoelectric field is channelled into Abrisokov flux tubes [5], in the same way as the ordinary magnetic field is in superconductors: the word dual indicates the interchange of roles between electric and magnetic fields and charges. The chromoelectric field mediating the force between coloured particles is squeezed by Meissner effect into flux tubes of constant energy per unit length, giving rise to the confining linear potential. These flux tubes behave as strings [3, 6]. The existence of strings in hadronic physics is supported by phenomenology [7,8]. They have also been visualized by numerical simulations of QCD on the lattice [9,10]. Some evidence in favour of dual superconductivity of the vacuum has been produced by Montecarlo simulations [11.

However a clear cut test of the mechanism is still missing. Such a test would be the detection of monopole condensation in the ground state, analogous to the condensation of Cooper pairs in the ground state of an ordinary superconductor. Condensation implies that the vacuum is a superposition of states with different charge, which, in turn, is nothing but a spontaneous breaking of the $U(1)$ symmetry related to charge conservation [12]. Such a breaking is signaled by a non-vanishing vacuum expectation value (vev) of any operator carrying non-trivial charge, as, e.g. the scalar field of the Landau-Ginzburg model of superconductivity. That vev is called a disorder parameter in the language of statistical mechanics.

In this paper, we give an explicit form of the creation operator of monopoles, together with a lattice version of it, in view of using its vev as a disorder parameter monitoring dual superconductivity. The operator is checked in $U(1)$ compact pure gauge theory on a lattice, where the behaviour of monopoles is known 13,14.

For $Q C D$, the strategy is the following. The monopoles which are expected to condense and generate superconductivity are Dirac monopoles of a residual $U(1)$ symmetry, which survives after a suitable gauge fixing, known as abelian projection [1]. An abelian projection is defined as the gauge transformation which diagonalizes any operator transforming in the adjoint representation of the gauge group. The points where two eigenvalues of that operator coincide correspond to singularities of the gauge transformation and have the topology of world-lines of point monopoles [1,15]. Such monopoles have been observed on the lattice [16-20]. Of course the location and the number of monopoles do depend on the choice of the operator used to define the abelian projection: a possibility, advocated by 't Hooft, is that physics, i.e. monopole condensation, is independent of it. The relevant abelian degrees of freedom can also be fixed by a somewhat different procedure, known as maximal abelian projection [15]. Our operator will allow to investigate unambigously what abelian projection, if any, defines the monopoles relevant to confinement.

In this paper, we will only present the construction of the operator and the way it works in lattice $U(1)$ compact gauge theory. For that theory, there exists a construction of a disorder variable describing monopole condensation [21], which is rigorous but based on a particular form of the action (the Villain action). Our operator coincides with the above one in the Villain case, but can be used with different forms of the action. This is particularly important in order to use it for non-abelian theories, where the effective $U(1)$ action after 
abelian projection is not known. The study of monopole condensation in $Q C D$ is in progress and will be presented elsewhere.

\section{THE MONOPOLE CREATION OPERATOR}

For the sake of definiteness, we shall consider here only the case of a $U(1)$ monopole. Our procedure can easily be generalized to all kind of solitons.

Let $B_{\mu}(\mathbf{x} ; \mathbf{y})=(0, \mathbf{b}(\mathbf{x}, \mathbf{y}))$ be the classical field produced in the location $\mathbf{x}$ by a Dirac monopole at rest in $\mathbf{y}$. We can make any choice for the gauge, e.g. by putting the string along the positive $z$-axis. Then, defining $\mathbf{r}=\mathbf{x}-\mathbf{y}$ and $r=\|\mathbf{r}\|$, we have:

$$
b_{i}(\mathbf{x}, \mathbf{y})=g \varepsilon_{3 i j} \frac{r_{j}}{r\left(r-r_{3}\right)}
$$

where $g$ is the charge of the monopole, satisfying the Dirac quantization condition:

$$
\text { e } g=\frac{n}{2}, n \text { integer }
$$

If $\Pi_{i}(\mathbf{x}, t)$ is the conjugate momentum to $A_{i}(\mathbf{x}, t)$, then the operator

$$
\mu(\mathbf{y}, t)=\exp \left\{i \int d^{3} \mathbf{x} b_{i}(\mathbf{x}, \mathbf{y}) \Pi_{i}(\mathbf{x}, t)\right\}
$$

creates a monopole at the location $\mathbf{y}$ and time $t$. This can be immediately seen in the Schrödinger representation of the fields, where we have:

$$
\mu(\mathbf{y}, 0)|\mathbf{A}(\mathbf{x}, 0)\rangle=|\mathbf{A}(\mathbf{x}, 0)+\mathbf{b}(\mathbf{x}, \mathbf{y})\rangle
$$

Equation (4) is a trivial consequence of the canonical commutation relations between the fields and their conjugate momenta, and is nothing but the field-theoretic equivalent of the familiar statement that $e^{i p a}$ translates the coordinate $q$ by $a$ :

$$
e^{i p a}|q\rangle=|q+a\rangle
$$

$\mu(\mathbf{y}, t)$, applied to any field configuration, adds a monopole to it.

This can be restated in terms of commutation relations as:

$$
\begin{aligned}
& {\left[A_{i}(\mathbf{x}, t), \mu(\mathbf{y}, t)\right]=b_{i}(x, y) \mu(\mathbf{y}, \mathbf{t})} \\
& {\left[\Pi_{i}(\mathbf{x}, t), \mu(\mathbf{y}, t)\right]=0}
\end{aligned}
$$

which also show that the electric field of the configuration is left unchanged. It is worthwhile to notice that the specific choice of the gauge for $\mathbf{b}$ is irrelevant: what really matters here is topology, which is independent of it. Our operator $\mu$ is similar to operators introduced in the literature by different constructions, in various contexts 22].

Now, if the ground state of the theory has a definite monopole number $N$, then, under a magnetic $U(1)$ rotation, we have: 


$$
\begin{aligned}
U|0\rangle & =e^{i \varphi N}|0\rangle \\
U \mu U^{\dagger} & =e^{i \varphi} \mu
\end{aligned}
$$

Hence:

$$
\langle 0|\mu| 0\rangle=\langle 0|\mu| 0\rangle e^{i \varphi}
$$

which implies:

$$
\langle 0|\mu| 0\rangle=0
$$

Therefore if

$$
\langle 0|\mu| 0\rangle \neq 0
$$

then $|0\rangle$ is not $U(1)$ invariant and there is spontaneous symmetry breaking of $U(1)$. A translation by a static field $\mathbf{g}(\mathbf{x})$ such that curl $\mathbf{g}=0$ in this language corresponds to a pure gauge transformation:

$$
\begin{aligned}
& \gamma(t)=\exp \left\{i \int d^{3} \mathbf{y} g_{i}(\mathbf{x}) \Pi_{i}(\mathbf{x}, t)\right\} \\
& \gamma(t)\left|A_{i}(\mathbf{x}, t)\right\rangle=\left|A_{i}(\mathbf{x}, t)+g_{i}(\mathbf{x})\right\rangle
\end{aligned}
$$

It is clear that $\gamma$ does not change $\mathbf{E}$ and, since $\mathbf{H}=$ curl $\mathbf{A}$, it does not change $\mathbf{H}$ either. Therefore, by gauge-invariance,

$$
\langle 0|\gamma(t)| 0\rangle=\langle 0 \mid 0\rangle=1
$$

Performing the Wick rotation to Euclidean space, we obtain:

$$
\mu_{E}(\mathbf{x}, t)=\exp \left\{-g \int d^{3} \mathbf{y} b_{i}(\mathbf{x}, \mathbf{y}) \Pi_{i}\left(\mathbf{x}, x_{4}\right)\right\}
$$

From now on, we will be interested only in the Euclidean quantity and drop for simplicity the $E$ subscript.

For free photons, rescaling the fields by a factor $1 / \sqrt{\beta}$ with $\beta=1 / e^{2}$,

$$
\langle\mu\rangle=\frac{\int \mathcal{D} A \exp \left\{-\beta \int\left[\frac{1}{4} F_{\mu \nu} F_{\mu \nu}+F_{0 i} b_{i}\right]\right\}}{\int \mathcal{D} A \exp \left\{-\beta \int\left[\frac{1}{4} F_{\mu \nu} F_{\mu \nu}\right]\right\}}
$$

The integral is Gaussian and can be directly computed giving:

$$
\langle\mu\rangle=\exp \left\{\frac{1}{2} \beta \int \frac{d^{4} k}{(2 \pi)^{4}}\left\langle F_{0 i}(k) F_{0 j}(k)\right\rangle b_{i}(\mathbf{k}) b_{j}(\mathbf{k})\right\}
$$

Since 


$$
\left\langle F_{0 i}(\mathbf{k}) F_{0 j}(\mathbf{k})\right\rangle=\frac{k_{4}^{2} \delta_{i j}+k_{i} k_{j}}{k^{2}}
$$

if $\mathbf{b}$ is such that $k_{i} b_{i}(k)=0$, we have

$$
\langle\mu\rangle=\exp \left\{\frac{\beta}{2} \int \frac{d^{4} k}{(2 \pi)^{4}}|\mathbf{b}(k)|^{2}-\frac{\beta}{4} \int \frac{d^{3} k}{(2 \pi)^{3}}|\mathbf{k}||\mathbf{b}(k)|^{2}\right\}
$$

The same calculation for a pure gauge transformation yields:

$$
\langle\gamma\rangle=\exp \left\{\frac{\beta}{2} \int \frac{d^{4} k}{(2 \pi)^{4}}|\mathbf{g}(k)|^{2}\right\}
$$

We realize that the first term in the exponent of eq.(20) is a normalization which can be subtracted by taking instead of $\langle\mu\rangle$ the ratio:

$$
\langle\bar{\mu}\rangle=\frac{\langle\mu\rangle}{\langle\gamma\rangle}
$$

where $\langle\gamma\rangle$ is defined by means of any gauge transformation $\mathbf{g}$, such that:

$$
\int \frac{d^{4} k}{(2 \pi)^{4}}|\mathbf{g}(k)|^{2}=\int \frac{d^{4} k}{(2 \pi)^{4}}|\mathbf{b}(k)|^{2}
$$

Then

$$
\langle\bar{\mu}\rangle=\exp \left\{-\frac{\beta}{4} \int \frac{d^{3} k}{(2 \pi)^{3}}|\mathbf{k}||\mathbf{b}(k)|^{2}\right\}
$$

The integral in the exponent, once regularized at small distances, tends to $+\infty$ as $V \rightarrow \infty$. In the infinite volume limit $\langle\bar{\mu}\rangle=0$ as it should be, since the perturbative vacuum has zero magnetic charge. The same situation appears when computing the overlap of the Fock vacuum to the Bogolubov rotated vacuum in a superconductor [23].

The same result is obtained if we work directly in the Schrödinger representation in the Coulomb gauge. There the vacuum wave functional is:

$$
\Psi(\Omega)=\prod_{\mathbf{k}} C_{\mathbf{k}} e^{-\frac{1}{2}|\mathbf{k}|\left|A_{\perp}(\mathbf{k})\right|^{2}}
$$

and

$$
\langle\Omega|\mu| \Omega\rangle=\exp \left\{-\frac{1}{4} \beta \int \frac{d^{3} k}{(2 \pi)^{3}}|\mathbf{k}||\mathbf{b}(k)|^{2}\right\}
$$




\section{LATTICE FORMULATION}

A lattice version of the operator $\mu$ is obtained by replacing $e F_{0 i}$ by the plaquette $P_{0 i}$, or better, by its imaginary part:

$$
e F_{0 i} \rightarrow \operatorname{Im} P_{0 i}
$$

and discretizing the field $b_{i}$.

Then the disorder parameter becomes:

$$
\langle\mu\rangle=\frac{\int \mathcal{D} U \exp \{-\beta S\} \exp \left\{-\frac{1}{2} \beta \sum_{n, i} b_{i}(n) \operatorname{Im} P_{0 i}(n)\right\}}{\int \mathcal{D} U \exp \{-\beta S\}}
$$

or, if we want to cancel the unwanted normalization, we can divide by:

$$
\langle\gamma\rangle=\frac{\int \mathcal{D} U \exp \{-\beta S\} \exp \left\{-\frac{1}{2} \beta \sum_{n, i} g_{i}(n) \operatorname{Im} P_{0 i}(n)\right\}}{\int \mathcal{D} U \exp \{-\beta S\}}
$$

obtaining

$$
\langle\bar{\mu}\rangle=\frac{\int \mathcal{D} U \exp \{-\beta S\} \exp \left\{-\frac{1}{2} \beta \sum_{n, i} b_{i}(n) \operatorname{Im} P_{0 i}(n)\right\}}{\int \mathcal{D} U \exp \{-\beta S\} \exp \left\{-\frac{1}{2} \beta \sum_{n, i} g_{i}(n) \operatorname{Im} P_{0 i}(n)\right\}}
$$

We stress once again that any gauge function $g_{i}$ is acceptable, provided the normalization condition (23) is satisfied.

If we blindly compute $\langle\mu\rangle$ or $\langle\bar{\mu}\rangle$ by numerical simulations, a first technical difficulty arises. We are faced with the usual problems encountered in computing quantities like a partition function, which are exponentials of extensive quantities, proportional to the number of degrees of freedom. The distribution of the values is not Gaussian and the error does not decrease by increasing statistics (see, e.g. [24], where the same problem appears in a different context). To avoid that, we will compute the quantity:

$$
\rho=\frac{d}{d \beta} \log \langle\bar{\mu}\rangle=\frac{d}{d \beta} \log \langle\mu\rangle-\frac{d}{d \beta} \log \langle\gamma\rangle
$$

At $\beta=0,\langle\mu\rangle=\langle\gamma\rangle=1$, and therefore:

$$
\log \langle\bar{\mu}\rangle=\int_{0}^{\beta} d \beta^{\prime} \rho\left(\beta^{\prime}\right)
$$

We easily get, putting

$$
\begin{aligned}
S_{b} & =\left.\frac{1}{2} \sum b_{i}(n) \operatorname{Im} P_{0 i}(n)\right|_{n_{0}=0} \\
S_{g} & =\left.\frac{1}{2} \sum g_{i}(n) \operatorname{Im} P_{0 i}\right|_{n_{0}=0}
\end{aligned}
$$


the following expression for $\rho$ :

$$
\rho=\left\langle S+S_{g}\right\rangle_{S+S_{g}}-\left\langle S+S_{b}\right\rangle_{S+S_{b}}
$$

which can be evaluated by numerical simulations. The subscript on the average indicates the action defining the Feynman integral.

The two quantities on the rhs have the same strong coupling expansion. Thus, the use of $\langle\bar{\mu}\rangle$ instead of $\langle\mu\rangle$, besides producing a cancellation of the spurious normalization of the Feynman path integral, can help in eliminating the lattice artefacts produced by the discretization which can spoil the continuum limit. This brings us to a second, more physical difficulty, which is the continuum limit. In fact, while for $Q C D$, which is asymptotically free, we expect that, at sufficiently high $\beta$, lattice artefacts should cancel, for a model like $U(1)$ this point is not so clear in principle. In Ref. [21] a proof is given of monopole condensation in the confined phase of $U(1)$, defining a disorder variable for the Villain action. We have checked that our $\langle\mu\rangle$ operator exactly coincides with the one of Ref. [21], when we use the Villain action: we expect that for Wilson action the same will hold. We have then computed numerically $\rho$.

For a good order parameter, we would expect $\rho$ to be zero, or $\langle\bar{\mu}\rangle=1$ below the critical value of the coupling $\beta_{c}$ and then to show a large negative peak around $\beta_{c}$, corresponding to a drop to zero of $\langle\bar{\mu}\rangle$. At larger values of $\beta$, we have free photons and Eq. (24) should hold.

Figure 1 shows the behaviour of $\rho$ for a $12^{4}$ lattice, for a monopole in the center of the space lattice. In order to be able to identify the signal as a genuine physical result (i.e. not due to lattice artefacts), we have performed a number of checks:

1) we have changed the form of $b_{i}$ by a gauge transformation to get the Wu-Yang expression of the monopole potential. The result does not change qualitatively.

2) $\langle\gamma\rangle$ shows practically no signal at $\beta_{c}$ within the errors, and does not change appreciably by changes of $g_{i}$. To test that we have computed $\langle\gamma\rangle$ for two different choices of $g_{i}(\mathbf{x})$, both satisfying the condition Eq.(23): $\left\langle\gamma_{1}\right\rangle$ for $\mathbf{g}_{1}(\mathbf{x})=$ const. and $\left\langle\gamma_{2}\right\rangle$ for $g_{2}(\mathbf{x}) \propto \mathbf{x} /|\mathbf{x}|^{2}$. In Fig.2 we display

$$
\rho_{\text {gauge }}=\frac{d}{d \beta} \ln \frac{\left\langle\gamma_{2}\right\rangle}{\left\langle\gamma_{1}\right\rangle}
$$

and $\rho=\frac{d}{d \beta} \ln \frac{\langle\mu\rangle}{\left\langle\gamma_{1}\right\rangle}$ for a $6^{4}$ lattice. $\rho_{\text {gauge }}$ shows no relevant signal at $\beta_{c}$.

3) We have measured the correlation function between a monopole antimonopole pair at large time distance, in the same position in space. To do that we define

$$
\begin{gathered}
C(d)=\langle\mu(\mathbf{0}, 0) \mu(\mathbf{0}, d)\rangle \\
S_{(b, \bar{b})}(d)=\left.\frac{1}{2} \sum b_{i}(n) \operatorname{Im} P_{0 i}(n)\right|_{n_{0}=0}-\left.\frac{1}{2} \sum b_{i}(n) \operatorname{Im} P_{0 i}(n)\right|_{n_{0}=d}
\end{gathered}
$$

and we measure 


$$
\rho_{(b, \bar{b})}(d)=2\left\langle S+S_{g}\right\rangle_{S+S_{g}}-\left\langle S+S_{(b, \bar{b})}(d)\right\rangle_{S+S_{(b, \bar{b})}(d)}
$$

Since

$$
\frac{d}{d \beta} \ln \frac{C(d)}{\langle\gamma\rangle^{2}}=\left.\rho_{(b, \bar{b})}(d) \quad C(d)\right|_{\beta=0}=1 \quad C(d)=\int_{0}^{\beta} \rho_{(b, \bar{b})}(d) d \beta
$$

By the cluster property we should have at large $d$ that $C(d) \rightarrow\langle\mu(\mathbf{0}, 0)\rangle^{2}$, or $\rho_{(b, \bar{b})}(d) \rightarrow$ $2 \rho$. Fig. 3 shows that this expectation is indeed verified.

We notice that the height of the negative peak of $\rho$ at $\beta_{c}$ increases with volume [see Fig.1 and Fig.2]. The value of $\beta_{c}$ as defined by the position of our peak is $\beta_{c}=1.01(1)$ for a $6^{4}$ lattice and $\beta_{c}=1.009(1)$ for a $12^{4}$ lattice.

We have taken monopole charge $n=4$ (Eq.(2)) to get a good signal with a relatively low statistics (tipically $10^{4}$ configurations per value of $\beta$ ): smaller charges $(n=1,2)$ give the same results but the signals are smaller and more noisy. Our statistical errors are shown in the figures, when they are larger than the symbols used.

\section{CONCLUSIONS}

The strong negative peak in $\rho$ at $\beta_{c}$ is a clear signal that monopoles are condensed in the confined phase, while they are not in the high- $\beta$ regime of the $U(1)$ model. The same signal can be extracted from $\langle\mu \bar{\mu}\rangle$ correlators. We expect a similar signal in $Q C D$ at the deconfining phase transition for the disorder variable creating $U(1)$ monopoles defined by abelian projection. Work is in progress and a comprehensive report will appear soon.

We underline that the technique used is exactly the same as for the $U(1)$ and that it can be extended straightforwardly to different systems in which the condensation of solitons can play a physical role, like the 3-d $X Y$ model, or the 4-d Georgi-Glashow model. Work is in progress also on these systems.

Acknowledgements: We thank M.Maggiore and S. Olejnik for collaboration in the early stages of this work. We thank M. Mintchev for useful discussions. 


\section{REFERENCES}

[1] G. 't Hooft, in "High Energy Physics", Proceedings of the EPS International Conference, Palermo 1975, ed. A. Zichichi, Editrice Compositori, Bologna 1976;

G. 't Hooft, Nucl Phys. B 190 (1981) 455.

[2] S. Mandelstam, Phys. Rep. 23C (1976) 245.

[3] H.B. Nielsen, P. Olesen, Nucl. Phys. B61 (1973) 45.

[4] For a review, see: A. Di Giacomo, Acta Phys. Polonica B25 (1994) 215.

[5] A.B. Abrikosov JETP 5 (1957) 1174.

[6] Y. Nambu Phys. Rev. D 10 (1974) 4262.

[7] G. Veneziano, Nuovo Cimento 57 A (1968) 190.

[8] C. Rebbi Phys. Rep. 12 C (1974) 1.

[9] A. Di Giacomo, M. Maggiore and Š. Olejník, Phys. Lett. B236 (1990) 199; Nucl. Phys. B347 (1990) 441.

[10] R.W. Haymaker, J. Wosiek, Phys. Rev. D 36 (1987) 3297.

[11] D.A. Brown, R.W. Haymaker, V. Singh, Phys. Rev. D 47 (1993) 1715

P. Cea, L. Cosmai, BARI preprint, to appear in the Proceeding of LATTICE 93.

[12] For a presentation of superconductivity in the language of high energy physicists, see: S. Weinberg, Progr. of Theor. Phys. Suppl. No. 86 (1986) 43.

[13] T. Banks, J. Kogut and R. Myerson, Nucl. Phys. B129 (1977) 493.

[14] T.A. DeGrand and D. Toussaint, Phys. Rev. D 22 (1980) 2478.

[15] A.S. Kronfeld, G. Schierholz, U.J. Wiese, Nucl. Phys. B293 (1987) 461.

[16] A.S. Kronfeld, M.L. Laursen, G. Schierholz, U.J. Wiese, Phys. Lett. B198 (1987) 516.

[17] T.L. Ivanenko, A.V. Pochinskii, M.I. Polikarpov, Phys. Lett. B 252 (1990) 252.

[18] L. Del Debbio, A. Di Giacomo, M. Maggiore, Š. Olejník, Phys. Lett. B267 (1991) 254.

[19] J. Polonyi, Nucl. Phys. A 461 (1987) 279.

[20] T. Suzuki, Nucl. Phys. B 30 (Proc. Suppl.) (1993) 176 and references therein.

[21] J. Fröhlich and P.A. Marchetti, Euro. Phys. Lett. 2 (1986) 933; Commun. Math. Phys. 112 (1987) 343; ibidem 116 (1988) 127; ibidem 121 (1989) 177; Lett. Math. Phys. 16 (1988) 347;

L. Polley and U. Wiese, Nucl. Phys. B356 (1991) 629.

[22] L.P. Kadanoff, H.Ceva, Phys. Rev. B3 (1971) 3918

E.C. Marino, B. Schroer, J.A. Swieca, Nucl. Phys. B 200 (1982) 473; A. Liguori, M. Mintchev, M. Rossi, Phys. Lett. B 305 (1993) 52.

[23] R. Haag, Il Nuovo Cimento XXV (1962) 287

[24] A. Hasenfratz, P. Hasenfratz, F. Niedermayer, Nucl. Phys. B 239, (1990) 739. 


\section{FIGURES}

FIG. 1. $\rho$ versus $\beta$ on a $12^{4}$ lattice

FIG. 2. $\rho$ and $\rho_{\text {gauge }}$ versus $\beta$ on a $6^{4}$ lattice.

FIG. 3. $\rho_{b \bar{b}}(d)$ versus $\beta$ at distances $d=4,7,9$, compared to $2 \rho$. 
This figure "fig1-1.png" is available in "png" format from: http://arxiv.org/ps/hep-lat/9403013v1 
This figure "fig1-2.png" is available in "png" format from: http://arxiv.org/ps/hep-lat/9403013v1 